\title{
Computational chemistry and drug discovery: a call to action
}

"Although program code is often shared within extended research groups or companies, it is rarely shared between groups.

This is in stark contrast to other fields, such as bioinformatics or statistics, in which the publication of source code and example datasets is required to accompany the published articles."

Keywords: collaboration $\approx$ computational chemistry $\|$ drug discovery $\approx$ education $\approx$ reproducibility $\approx$ Teach-Discover-Treat

The practitioners of computational chemistry devoted to drug discovery use computational approaches to unravel complex relationships in chemical and biological datasets. They also use computational tools to design chemical matter using intricate multi-objective optimization methods. In both applications, the discipline formulates hypotheses that inspire a next round of experiments. The impact from these approaches is critically dependent on productive relationships across multiple disciplines for generating and annotating data, as well as for the required follow-up on the hypotheses.

Numerous examples of drugs that have been discovered and optimized with contributions from computational chemists exist [1]. Computational methods provide guidance but are by no means able to make perfect predictions. It is, therefore, important that we share details of our work so that as a community we can learn from what works, as well as what fails. To increase the impact of our computational drug-discovery efforts, we present a call to action to our scientific community.

This call to action is embodied by the TeachDiscover-Treat (TDT) initiative $[2,101]$. Our ultimate goals include strengthening the education of drug-discovery scientists across disciplines and increasing collaboration and scientific rigor within the computational chemistry discipline.

\section{Drug discovery as a collaborative effort}

Two aspects of collaborative drug discovery deserve special attention in light of this call to action. The first is that the multidisciplinary workforce needs to have an understanding of the impact, reach and limitations of computational chemistry with respect to various discovery activities. This necessitates access to quality teaching materials for undergraduate and graduate courses, including case studies, and access to datasets and computational tools that are state-of-the-art.

The second aspect relates to the novel types of partnerships that are being formed and explored between entities in the public, private, nonprofit and government sectors. In January 2012, 13 pharmaceutical companies, the USA, UK and UAE governments, the Bill \& Melinda Gates Foundation, the World Bank and other global health organizations committed to working together to eradicate or control ten neglected tropical diseases by 2020 [102]. A notable collaboration focused on antimalarials involves GlaxoSmithKline, Novartis and St. Jude, who have all made data from their whole cell screening campaigns publicly available $[3,103]$. A number of initiatives extend the open innovation or crowd sourcing concept beyond neglected diseases with efforts to extend target and compound spaces [4-6].

To maximize impact in complex discovery environments with many different partners, computational scientists need access to standardized data formats, annotated experimental data (for actives and inactives), and validated computational methods and models in formats that allow collaboration. With respect to standardized data formats, the community seems to have agreed on SMILES and MDL Mol or SD files as a standard means of distribution of chemical structure information. Providing access to annotated data is a challenging, active area in the drug-discovery community with a number of efforts that provide open access (ChEMBL [103], CDD [104] and PubChem [105]). However, the availability of datasets from 'real world' discovery efforts beyond screening is still very limited.

\section{Striving for scientific excellence}

While computational chemistry and cheminformatics have made significant advances over

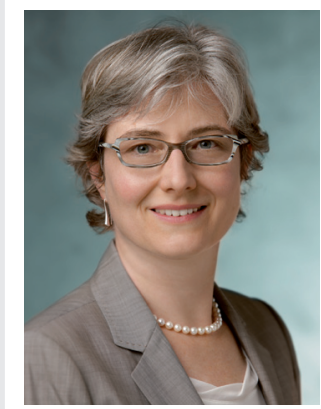

Johanna M Jansen

Author for correspondence: Novartis Institutes for Biomedical Research, Global Discovery Chemistry/Oncology, Infectious Diseases, \& Exploratory Chemistry, Emeryville, CA 94608, USA E-mail: johanna.jansen@ novartis.com

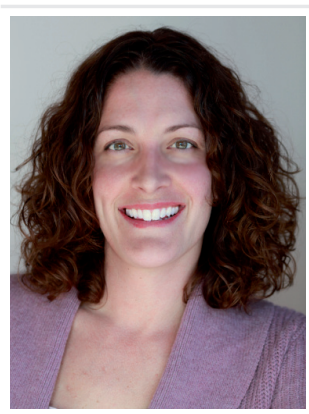

Rommie E Amaro

Department of Chemistry \& Biochemistry, University of California, San Diego, La Jolla, CA 92093, USA

Authors' affiliations continued overleaf... 


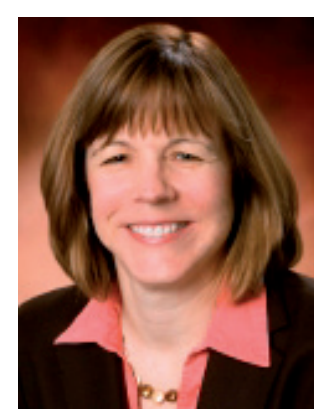

Wendy Cornell

Merck Research Labs, Knowledge Discovery/Knowledge Management, Rahway, NJ 07065, USA

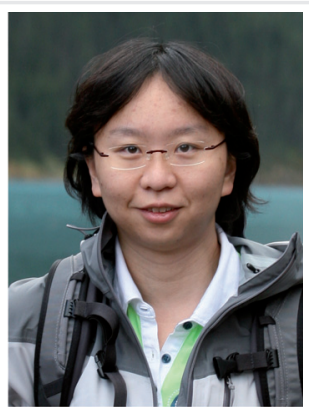

Y Jane Tseng

Graduate Institute of Biomedical Electronics \& Bioinformatics, National Taiwan University, Taipei, Taiwan

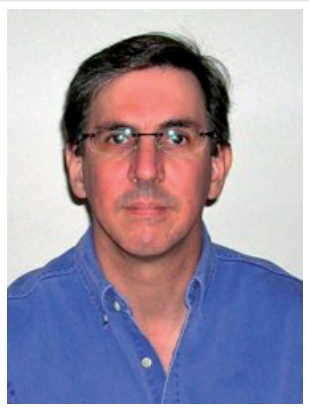

W Patrick Walters

Vertex Pharmaceuticals Inc., Cambridge, MA 02139, USA the past 40 years, much of this work has been carried out in isolation. As a result of this isolation, our field has evolved numerous versions of programs, both commercial and academic, which perform the same function. As an example, there are easily more than a dozen widely used programs that dock small molecules into a protein binding site. These programs differ primarily in the scoring function used to evaluate the interaction, the sampling method used to position the small molecule or both. Numerous studies have compared these programs and found that the overall performance of most is roughly equivalent [7-11]. One wonders if cooperation would have led to further, and more rapid, advances in the field.

In the early days of computational chemistry the Quantum Chemistry Program Exchange was established to share subroutines for quantum chemistry with contributions from both academic and industrial members [12] More recently, open source platforms such as SourceForge [106] have become a depository of relevant computational tools, including open source toolkits for computational chemistry and cheminformatics. Software packages such as Open Babel [107], CDK [108], RDKit [109] and Indigo [110] are now freely available for download from the internet. While these toolkits provide a starting point for developing new applications, the learning curve for most is rather steep. In addition, many of these toolkits lack documentation and simple example programs that can provide the basis for future development. This situation is improving with the recent publication of books documenting these toolkits [111-113] and a website [114] that provides comparative examples using multiple toolkits. While these toolkits are widely available, there are few publications that feature their use in the development of new methods. Hopefully a move toward more reproducible research would spur an increased interest in these toolkits.

Related to these ideas is the undeniable fact that, as a community, we need to change the way we publish [13]. Many groups have published on the development of new methods and application to specific systems, but very few of these publications have included program source code or data. Although program code is often shared within extended research groups or companies, it is rarely shared between groups. This is in stark contrast to other fields, such as bioinformatics or statistics, in which the publication of source code and example datasets is required to accompany the published articles. While publications sometimes contain sufficient detail to allow computational methodology to be reproduced, without access to the original code, it is difficult to determine whether a method has been correctly implemented. The ability to reproduce a model and/ or methodology is critically dependent on having access to utilized program codes (including specific builds or versions), structures (datasets) and associated input files. Although changing the publication requirements across all possible scientific journals is a daunting task, we can take action as a community: as authors, we can choose to submit this information as supporting information; as reviewers, we can request that authors submit this information as a prerequisite to review and subsequent publication. The fact that innovative new journal formats, which allow the accompanying article 'metadata' to be managed alongside the research articles, have been so well received (e.g., consider the PLoS journals [115]) is a promising indication that such publication ideals are not in fact daunting at all.

\section{"The Teach-Discover-Treat initiative provides one model for generating and disseminating drug-discovery workflows. We anticipate that this initiative will spawn other efforts, bringing new perspectives and methods into the field of drug discovery."}

In addition to making it easier for others to reproduce methodology, we need to make 'real world' datasets widely available that cover all aspects of computational drug discovery. An effort to acknowledge in this area is Community Structure-Activity Resource (CSAR), which aims to provide the scientific community with the highest quality data for a diverse collection of proteins and small molecule ligands to train scoring functions and develop new docking algorithms [14]. The availability of real world datasets will make it much easier to perform head-to-head comparisons and quantitate improvements to existing methods. To prevent code from being overly optimized to fit the available data sets, new data sets must be provided on a regular basis to objectively assess method and code performance. Open datasets also allow both new and experienced users to experiment and gain a more thorough understanding of 
molecular interactions. These datasets can also make it easier to recruit scientists from other fields who may be able to bring new perspectives. One of the best sources of these data is pharmaceutical drug discovery efforts. While management and intellectual property groups in industry are sometimes reluctant to release proprietary data, there are often completed or abandoned programs for which data can be released. Those working in industry need to persist in working to find new datasets that can be released, and can potentially lead to improvements in the field.

\section{The TDT initiative}

The TDT initiative was launched to help address these challenges, while fueling innovative drugdiscovery approaches against targets in neglected diseases, such as malaria and African sleeping sickness. We seek to motivate the community within the framework of a four-category competition involving the development of drugdiscovery workflows around the topics of highthroughput screening, novel chemotypes, lead optimization and broadly defined innovation. Submissions must develop outstanding workflows using freely available software, to enable universal access. To amplify the impact of the models, innovative, clear and thorough 'tutorials' are required to be submitted alongside the workflows. Both the workflow itself and associated tutorial are part of the judging criteria [116]. Real world impact will be realized for the winning submissions through strategic partnerships enabling compound acquisition, medicinal chemistry synthesis and biochemical assays. Winners will have the opportunity to further engage the community by presenting their workflows at the American Chemical Society national meeting in New Orleans, USA, in the spring of 2013 .

\section{"We have entered an era in which large amounts of CPU power are readily available and modeling software has become much easier to use."}

Computational drug discovery has evolved considerably over the past 40 years. We have entered an era in which large amounts of CPU power are readily available and modeling software has become much easier to use. Simultaneously, the internet has facilitated the creation of a global scientific community where most of the barriers to collaboration no longer exist. As methods that were once the domain of experts become available to a wider community, it is important that tutorials and training materials are available to educate interested scientists. The TDT initiative provides one model for generating and disseminating drug-discovery workflows. We anticipate that this initiative will spawn other efforts, bringing new perspectives and methods into the field of drug discovery. It is our hope that efforts such as TDT will benefit the drug-discovery community and ultimately help patients.

\section{Financial \& competing interests disclosure}

RE Amaro is supported in part by the National Institutes of Health through the NIH Director's New Innovator Award Program DP2-OD007237. The authors have no other relevant affliations or financial involvement with any organization or entity with a financial interest in or financial conflict with the subject matter or materials discussed in the manuscript apart from those disclosed.

No writing assistance was utilized in the production of this manuscript.

\section{References}

1 Jorgensen WL. The many roles of computation in drug discovery. Science 303(5665), 1813-1818 (2004).

2 Jansen JM, Cornell W, Tseng YJ, Amaro RE. Teach-Discover-Treat (TDT): collaborative drug discovery for neglected diseases. J. Mol. Graph. Model. doi: 10.1016/j. jmgm.2012.07.007 (2012) (In Press).

3 Guiguemde WA, Shelat AA, Garcia-Bustos JF, Diagana TT, Gamo FJ, Guy RK. Global phenotypic screening for antimalarials. Chem. Biol. 19(1), 116-129 (2012).

4 Lessl M, Bryans JS, Richards D, Asadullah K. Crowd sourcing in drug discovery. Nat. Rev. Drug Discov. 10(4), 241-242 (2011).
5 Norman TC, Bountra C, Edwards AM, Yamamoto KR, Friend SH. Leveraging crowdsourcing to facilitate the discovery of new medicines. Sci. Transl. Med. 3(88), 88mrl (2011).

6 Strauss S. Pharma embraces open source models. Nat. Biotechnol. 28(7), 631-634 (2010).

7 Cross JB, Thompson DC, Rai BK et al. Comparison of several molecular docking programs: pose prediction and virtual screening accuracy. J. Chem. Inf. Model. 49(6), 1455-1474 (2009).

8 McGaughey GB, Sheridan RP, Bayly CI et al. Comparison of topological, shape, and docking methods in virtual screening.
J.Chem. Inf. Model. 47(4), 1504-1519 (2007).

9 Cummings MD, DesJarlais RL, Gibbs AC, Mohan V, Jaeger EP. Comparison of automated docking programs as virtual screening tools. J. Med.Chem. 48(4), 962-976 (2005).

10 Perola E, Walters WP, Charifson PS. A detailed comparison of current docking and scoring methods on systems of pharmaceutical relevance. Proteins 56(2), 235-249 (2004).

11 Cornell WD. Recent evaluations of high throughput docking methods for pharmaceutical lead finding - consensus and caveats. Annu. Rep. Comput. Chem. 2, 297-323 (2006). 
12 Boyd DR. How computational chemistry became important in the pharmaceutical industry. In: Reviews in Computational Chemistry. Lipkowitz DB, Cundari TR (Eds). John Wiley and Sons, Inc., London, UK, 401-451 (2007).

13 Bourne PE. What do I want from the publisher of the future? PLoS Comput.Biol. 6(5), e1000787 (2010).

14 Dunbar JB Jr, Smith RD, Yang CY et al. CSAR benchmark exercise of 2010: selection of the protein-ligand complexes. J. Chem. Inf. Model. 51(9), 2036-2046 (2011).

\section{- Websites}

101 Teach-Discover-Treat. www.teach-discover-treat.org

102 Bill and Melinda Gates foundation. Private and Public Partners Unite to Combat 10 Neglected Tropical Diseases by 2020 . www.gatesfoundation.org/press-releases/pages/ combating-10-neglected-tropicaldiseases-120130.aspx
103 ChEMBL database. www.ebi.ac.uk/chembldb

104 Collaborative drug discovery. www.collaborativedrug.com

105 PubChem. http://pubchem.ncbi.nlm.nih.gov

106 Source Forge. http://sourceforge.net

107 Open Babel. http://openbabel.org

108 Chemistry Development Kit. http://sourceforge.net/apps/mediawiki/cdk

109 RDKit. Cheminformatics and Machine Learning Software. www.rdkit.org

110 Indigo toolkit. http://ggasoftware.com/opensource/indigo

111 Groovy cheminformatics with the chemistry development kit. www.lulu.com/shop/egon-willighagen/ groovy-cheminformatics-with-the-chemistrydevelopment-kit/paperback/ product-20267607.html
112 How to use Jmol to study and present molecular structures.

http://books.google.com/ books?id=59kQFyfbsJMC

113 Open Babel. Official user guide. www.lulu.com/shop/open-babel-developers/ open-babel/paperback/ product-14927363.html

114 Chemistry Toolkit Rosetta Wiki. http://ctr.wikia.com/wiki/Chemistry_ Toolkit_Rosetta_Wiki

115 PLoS journals. www.plos.org/publications/journals

116 Teach-Discover-Treat. Competition details. www.teach-discover-treat.org/details-for-thecompetition 